Revue des patrimoines

29 | 2016

Ensembles mobiliers, industriels, techniques.

Connaissance, protection, conservation, présentation au public

\title{
Ensembles mobiliers, industriels, techniques. Connaissance, protection, conservation, présentation au public ÉDITORIAL
}

Judith Kagan, Dominique Séréna-Allier et Anne Tricaud

\section{(2) OpenEdition \\ Journals}

Édition électronique

URL : http://journals.openedition.org/insitu/13500

DOI : 10.4000/insitu. 13500

ISSN : 1630-7305

Éditeur

Ministère de la Culture

Référence électronique

Judith Kagan, Dominique Séréna-Allier et Anne Tricaud, « Ensembles mobiliers, industriels, techniques. Connaissance, protection, conservation, présentation au public », In Situ [En ligne], 29 | 2016, mis en ligne le 28 août 2017, consulté le 24 septembre 2020. URL : http://journals.openedition.org/insitu/ 13500 ; DOI : https://doi.org/10.4000/insitu.13500

Ce document a été généré automatiquement le 24 septembre 2020.

\section{(c) (i) () $\Theta$}

In Situ Revues des patrimoines est mis à disposition selon les termes de la licence Creative Commons Attribution - Pas d'Utilisation Commerciale - Pas de Modification 4.0 International. 


\section{Ensembles mobiliers, industriels, techniques. Connaissance, protection, conservation, présentation au public} ÉDITORIAL

Judith Kagan, Dominique Séréna-Allier et Anne Tricaud

Car, en fin de compte, le but d'un ameublement

harmonieux est bien, comme on vient de le montrer, de refléter l'homme, mais de le refléter dans son essence idéale, ce qui est une exaltation

du moi.

C'est pourquoi, plus encore peut-être que la peinture, la sculpture, et même l'architecture, le mobilier révèle l'esprit d'une époque... Mario Praz. Histoire de la décoration d'intérieur : La philosophie de l'ameublement [1964], trad. fr. Londres/Paris: Thames et Hudson, 1994, p. 20.

Le 7 juillet 2016 a été promulguée la loi relative à la liberté de création, à l'architecture et au patrimoine (LCAP), portée depuis 2012 par le ministère de la Culture et de la Communication. Cette loi comprend plusieurs dispositions insistant sur la notion d'ensemble et celle de cohérence, qu'elle soit d'ordre historique, artistique, scientifique, archéologique ou architectural. La loi évoque ces notions tant pour les trésors nationaux, les archives, les biens mobiliers archéologiques ou les objets mobiliers que pour les domaines nationaux ou les sites patrimoniaux remarquables. Dans le domaine des monuments historiques, de nouvelles dispositions, attendues depuis longtemps, permettent le classement au titre des monuments historiques d'ensembles mobiliers et la création d'une servitude de maintien dans les lieux de certains objets classés au titre des monuments historiques dans un immeuble classé. 

propositions des parlementaires mais désormais, l'entrée en vigueur de ces dispositions est immédiate et il est possible de proposer ces nouvelles mesures de protection, avec l'accord des propriétaires.

\section{Articles du Code du patrimoine}


Les nouvelles dispositions du code du patrimoine :

«Art. L. 622-1-1. - Un ensemble ou une collection d'objets mobiliers dont la conservation dans son intégrité et sa cohérence présente un intérêt public au point de vue de l'histoire, de l'art, de l'architecture, de l'archéologie, de l'ethnologie, de la science ou de la technique peut être classé au titre des monuments historiques comme ensemble historique mobilier par décision de l'autorité administrative, après avis de la Commission nationale du patrimoine et de l'architecture.

"Cet ensemble ne peut être divisé ou aliéné par lot ou pièce sans autorisation de cette autorité.

«Les effets du classement s'appliquent à chaque élément de l'ensemble historique mobilier classé et subsistent pour un élément s'il est dissocié de l'ensemble. Toutefois, lorsque l'élément dissocié ne bénéficie pas d'un classement en application de l'article L. 622-1, les effets du classement peuvent être levés pour cet élément par l'autorité administrative.

« Art. L. 622-1-2. - Lorsque des objets mobiliers classés ou un ensemble historique mobilier classé sont attachés, par des liens historiques ou artistiques remarquables, à un immeuble classé et forment avec lui un ensemble d'une qualité et d'une cohérence dont la conservation dans son intégrité présente un intérêt public, ces objets mobiliers ou cet ensemble historique mobilier peuvent être grevés d'une servitude de maintien dans les lieux par décision de l'autorité administrative, après avis de la Commission nationale du patrimoine et de l'architecture et accord du propriétaire. Cette servitude peut être levée dans les mêmes conditions. En cas de refus de l'autorité administrative de lever la servitude, les sujétions anormales qui peuvent en résulter sont compensées par une indemnité. À défaut d'accord amiable, l'action en indemnité est portée devant le juge judiciaire.

"Le déplacement de cet objet mobilier ou de tout ou partie de cet ensemble historique mobilier classé est subordonné à une autorisation de l'autorité administrative.

«La servitude de maintien dans les lieux peut être prononcée en même temps que la décision de classement des objets mobiliers ou de l'ensemble historique mobilier, ou postérieurement à celle-ci. »

En 2014, au cours de la gestation du projet de loi, il nous a paru intéressant de faire le point sur ce que recouvre cette notion d'ensemble mobilier dans tous les domaines patrimoniaux, tant du point de vue de l'identification et de la protection juridique que de la conservation et de la transmission. En juin 2014, la revue In Situ a lancé un appel à contributions en vue de rassembler des articles permettant d'approfondir la compréhension du processus de création, de production et de conservation d'un ensemble, que celui-ci soit porteur de la mémoire d'un homme, d'une famille, d'une institution publique ou d'une communauté professionnelle, et qu'il soit ou non lié historiquement au lieu qui l'abrite. 
Figure 1

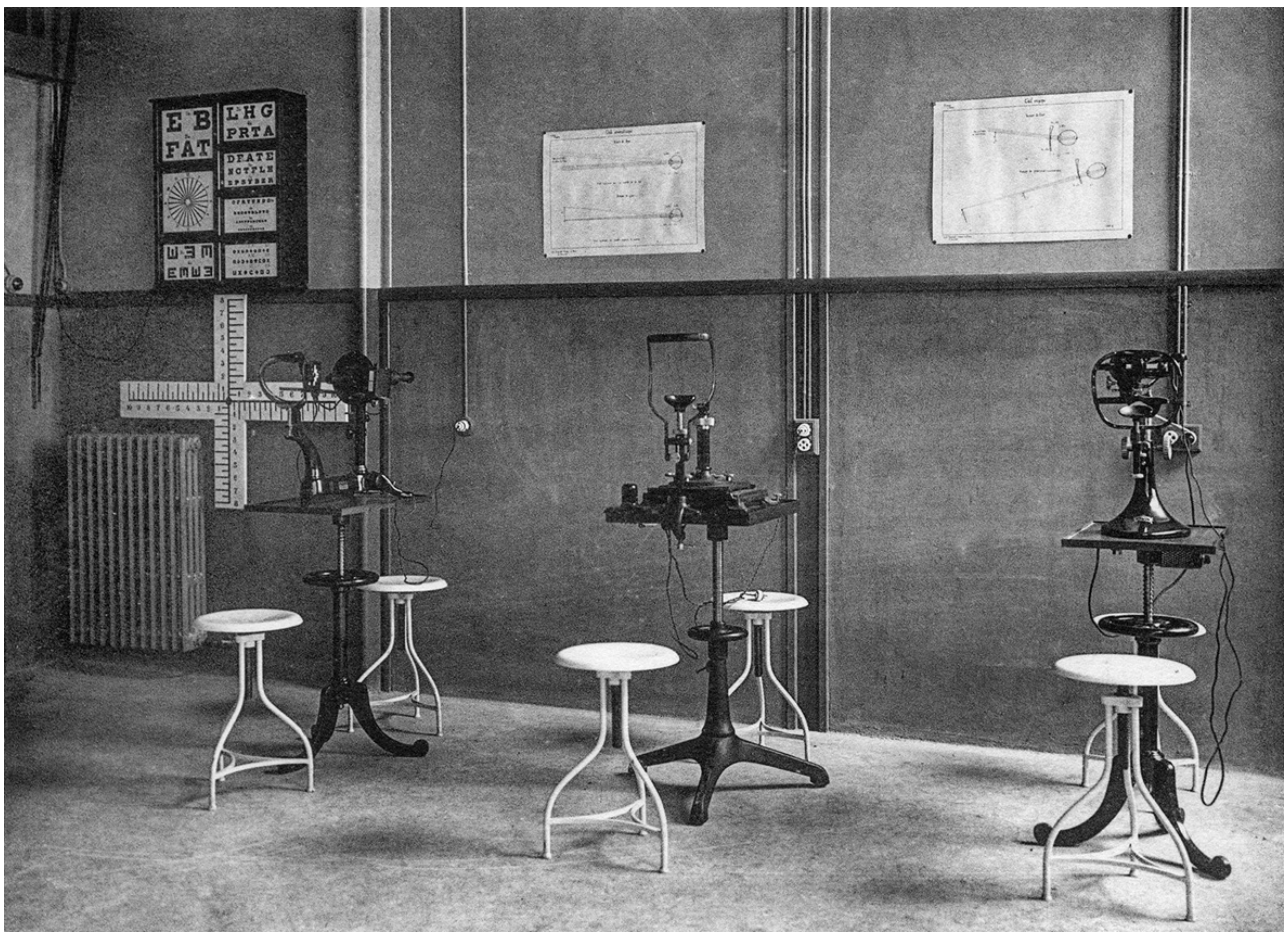

Lycée polyvalent Victor Bérard : Laboratoire d'Optométrie, décennies 1930-1940. Photographie imprimée, s.n. (Coll. lycée polyvalent Victor Bérard, Morez).

Repro. Sancey, Yves. @ Région Franche-Comté, Inventaire du Patrimoine, ADAGP, 2010. Base Mémoire : IVR43_20103900694NUC2A_P.

9 Notre appel a été largement entendu puisque plus de 50 propositions ont été reçues entre juin et octobre 2014 et près de 30 articles ont été rendus, traitant de récentes identifications dans le cadre de l'Inventaire général du patrimoine culturel, de protections au titre des monuments historiques, des Musées de France ou d'acquisitions d'ensembles mobiliers réintégrant leur édifice d'origine.

Ce numéro présente des cas exemplaires de conception homogène entre architecture et meubles, que ceux-ci soient meubles par nature ou pleinement intégrés à l'édifice et ce, quel que soit l'usage premier de l'édifice. Ses articles concernent aussi bien le patrimoine civil (demeures en ville ou aux champs), le patrimoine scientifique et technique (usines ou ateliers, lieux de la recherche) que le patrimoine religieux (lieux de culte en service actifs ou non).

11 Ils sont répartis selon trois axes, même si la frontière est parfois ténue entre les différents thèmes :

12 - connaissance et protection

13 - conservation et présentation au public

14 - échecs et reconquêtes.

15 La plupart de ces essais ont trait à des ensembles maintenus ou restitués dans le lieu pour lequel ils ont été créés. Certains exemples rendent compte d'ensembles historiquement liés mais transplantés dans un autre lieu, comme les ensembles écologiques de l'ancien musée des Arts et Traditions populaires, ou de la reconstitution à partir de sources d'archives d'ensembles d'art décoratif permettant de remeubler les châteaux, avec deux exemples suisses, qui revitalisent le principe des "period rooms». 
Les modalités d'inventaire, de conservation et de présentation des « dioramas » sont évoquées à travers le cas du Museon Arlaten.

Figure 2

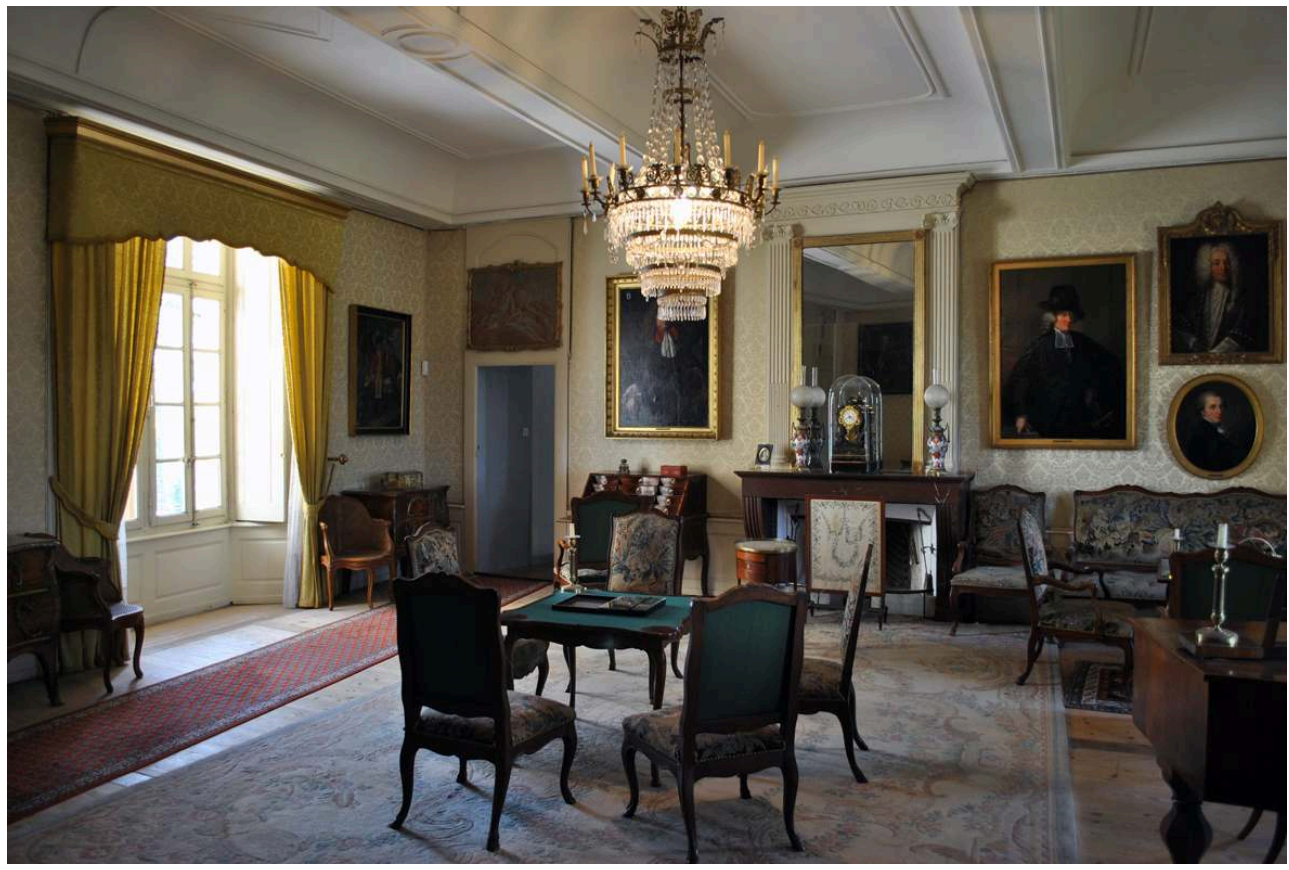

Le château de la Sarraz, Suisse. Le grand salon dans son état récent avec le mobilier attribuable à l'ébéniste Matthäus Funk, conservé dans cette pièce depuis près de deux siècles.

Phot. Rutz, Nicolas Alexandre. (c) Château de La Sarraz, Musée romand, 2012.

\section{Connaissance et protection}

Tout d'abord, une dizaine d'articles évoquent cette notion d'ensembles mobiliers à travers des exemples variés concentrés sur un seul site, sur une typologie ou sur un territoire : mobilier de château, collection familiale auquel il convient de donner un sens et une présentation muséale, lieu de mémoire d'un artiste en Corse, matériel scientifique rare comme le générateur Cockroft-Walton ou matériel d'enseignement d'optique et d'horlogerie, réflexion à l'échelle d'un ensemble technique similaire (les scieries vosgiennes) ou d'une région telle que la Bourgogne... Plusieurs exemples montrent comment assurer la survie d'un ensemble scientifique ou technique dont l'usage est obsolète dans la communauté qui l'a produit. 
Figure 3

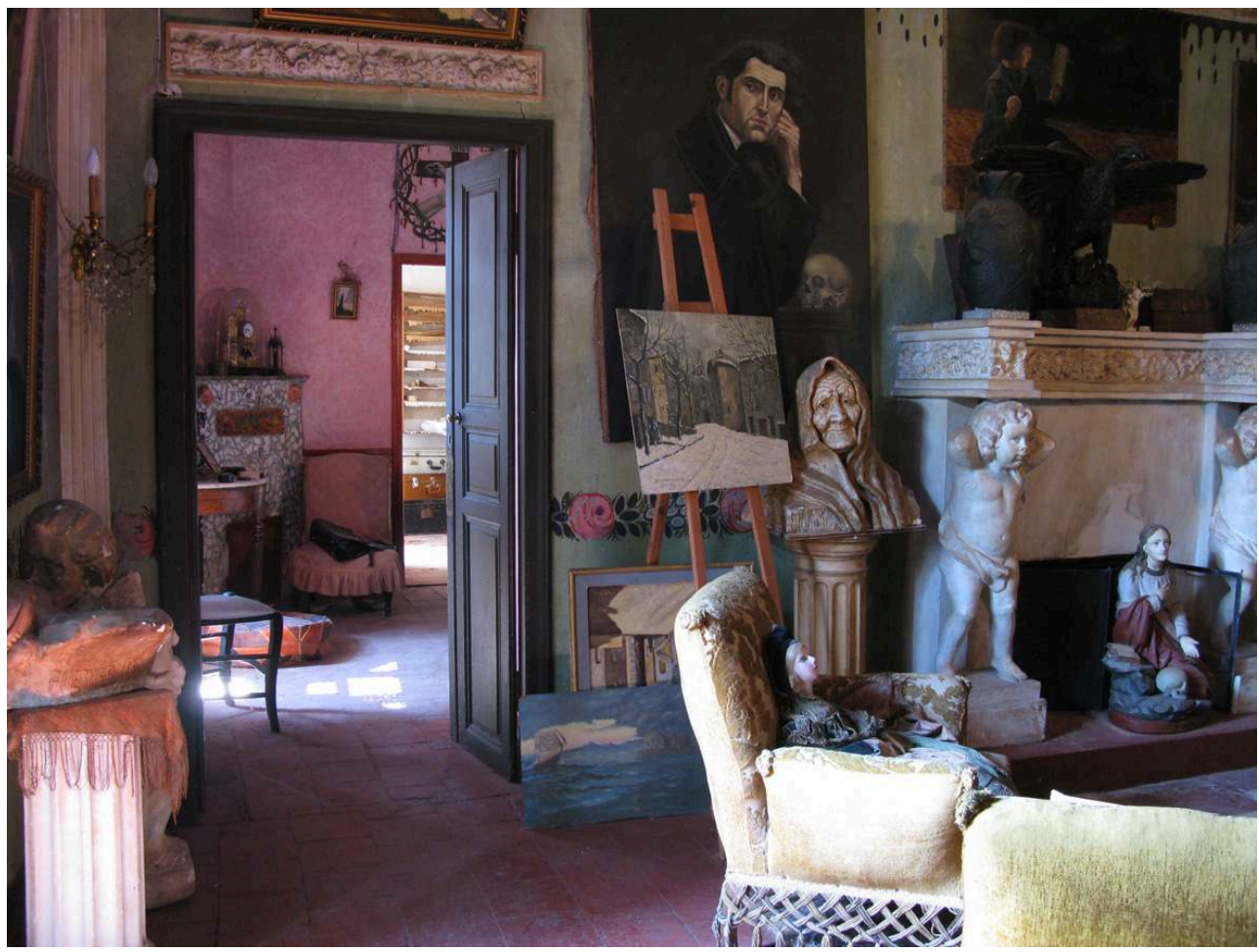

Maison Maestracci, Occhiatana, Haute-Corse. Salon, vue intérieure.

Phot. Tristani, Julia. (C) Collectivité territoriale de Corse, musée de la Corse, 2010.

17 Les réponses apportées varient mais deux cas de protection de bâtiments religieux pris dans leur ensemble, murs et mobilier, sont particulièrement remarquables. Il s'agit de l'église Saint-Nicaise de Reims (Marne), œuvre de l'architecte Auburtin, propriété d'un office HLM et mise à disposition de l'association diocésaine, décorée par de nombreux artistes dont Maurice Denis entre 1925 et 1933 d'une part, et de la chapelle SainteMadeleine de Bourg-en-Bresse, «chef-d'œuvre de l'Art déco dans l'Ain » d'autre part. On voit dans ces deux exemples tout l'intérêt que pourront avoir les nouvelles dispositions législatives reconnaissant la notion d'ensemble et consacrant le maintien dans les lieux. 
Figure 4

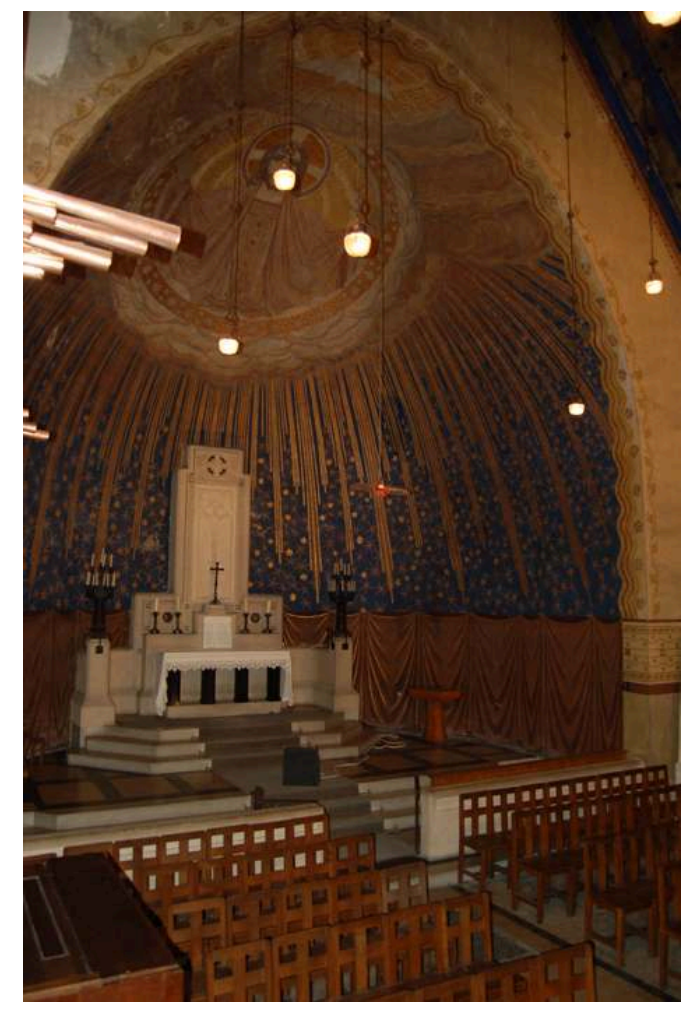

Intérieur de l'église Saint-Nicaise, Reims. Vue vers le chœur. Peintures de Gustave Jaulmes, assisté d'Henri Menu, 1924.

Phot. CRMH Champagne-Ardenne. (C) CRMH Champagne-Ardenne.

Nous n'avons reçu aucune proposition d'article relative aux tribunaux où pourtant, l'évolution contemporaine de l'usage est au cœur de la problématique. La fermeture des tribunaux et leur changement d'usage ont dispersé bien des aménagements cohérents. Il est aussi parfois difficile de concilier dans les établissements de santé présence des malades ou des résidents et préservation du patrimoine artistique. Ce thème sera plus précisément traité dans un prochain numéro de la revue In Situ consacré aux «patrimoines de la santé : essais de définition - enjeux de conservation $»^{2}$. Un dernier regret : nous ne publions aucune contribution concernant l'archéologie ou les archives, à l'exception de l'exemple du traitement du fonds Francis Jourdain, entré au musée d'Art et d'Histoire de Saint-Denis (Seine-Saint-Denis) ou celui des archives Flammarion à Juvisy-sur-Orge (Essonne).

\section{Conservation et présentation au public}

19 En ralliant le champ patrimonial, les ensembles mobiliers industriels ou techniques in situ posent aussitôt à leurs gestionnaires des problèmes complexes de conservation.

Composés d'éléments souvent hétérogènes en raison de leur échelle ou de leurs matériaux constitutifs, ils se révèlent, dans la majorité des cas, indissociables de leur environnement architectural, paysager ou domestique initial et participent, dans une localisation rarement interchangeable à l'histoire, à l'image des lieux. Aussi, au-delà des restaurations nécessaires, leur maintien sur place ne peut faire l'impasse sur l'unicité de l'ensemble et sur les interactions manifestes avec les lieux qui les abritent, 
parfois disséminés sur tout un territoire et peu compatibles avec la notion de préservation. Il faut souvent, en outre, intégrer la présence du public qui déambule dans un espace restreint réglementairement inadapté à la visite mais entend disposer des informations liées à ces ensembles dans une présentation la plus discrète possible. En croisant ces logiques parfois contradictoires, la démarche de conservation préventive est à même de relever ce défi. Elle permet en effet de trouver, dans un équilibre toujours précaire mais par une approche globale, des solutions pragmatiques adaptées à chaque cas ; elle est cependant rendue plus complexe lorsque les éléments composant un ensemble disposent d'un statut légal différencié selon qu'ils sont protégés au titre des monuments historiques, inscrits à l'inventaire réglementaire d'un musée ou d'un fonds d'archives ou qu'ils s'apparentent à un simple décor, un environnement paysager, à un document ou à une collection d'étude.

Les auteurs participant à ce numéro inventorient, dans une déclinaison passionnante, les mesures et les interventions conservatoires, souvent ingénieuses, qui ont été mises en œuvre pour préserver ces ensembles de l'usure du temps et des dégradations dues au public et construisent en creux un mode opératoire éventuellement modélisable.

Dans la majorité des exemples, la prise en compte de l'environnement propre à chaque ensemble se révèle la démarche la mieux partagée. Pour autant, une régulation climatique conforme aux normes est rarement envisageable puisqu'elle se heurte à des localisations problématiques, celles par exemple des scieries vosgiennes implantées près de cours d'eau, mais aussi à l'échelle de certains bâtiments, comme à l'église SaintNicaise de Reims, ou à l'exigüité et à la complexité de certains lieux comme celui où a vécu et travaillé Camille Flammarion à Juvisy-sur-Orge.

24 Au-delà de la rotation classique des objets pratiquée de manière systématique dans les musées ou les lieux historiques, certaines mesures compensatoires s'avèrent efficaces pour limiter les effets néfastes d'un milieu hostile. Ainsi, les chocs climatiques qu'occasionnerait le transfert, même temporaire, du trône de Napoléon $\mathrm{I}^{\text {er }}$ présenté au Sénat depuis deux siècles dans un environnement non conforme mais stable ont été évités par la suspension de tout prêt.

De même, les dégradations récurrentes des bâtiments à pans de bois des scieries vosgiennes ont été ralenties grâce à un entretien régulier et approfondi qui va au-delà de celui généralement préconisé.

Dans cette même perspective, dans la maison du peintre Foujita à Villiers-le-Bâcle (Essonne) ou à la chapelle des Pénitents blancs de Villeneuve-sur-Lot (Lot-et-Garonne), alors qu'il se révélait impossible de limiter par des enveloppes étanches les contaminations biologiques liées à un empoussièrement important, des campagnes de nettoyage intensif et plus fréquentes qu'à l'ordinaire ont été programmées pour assainissement. 
Figure 5

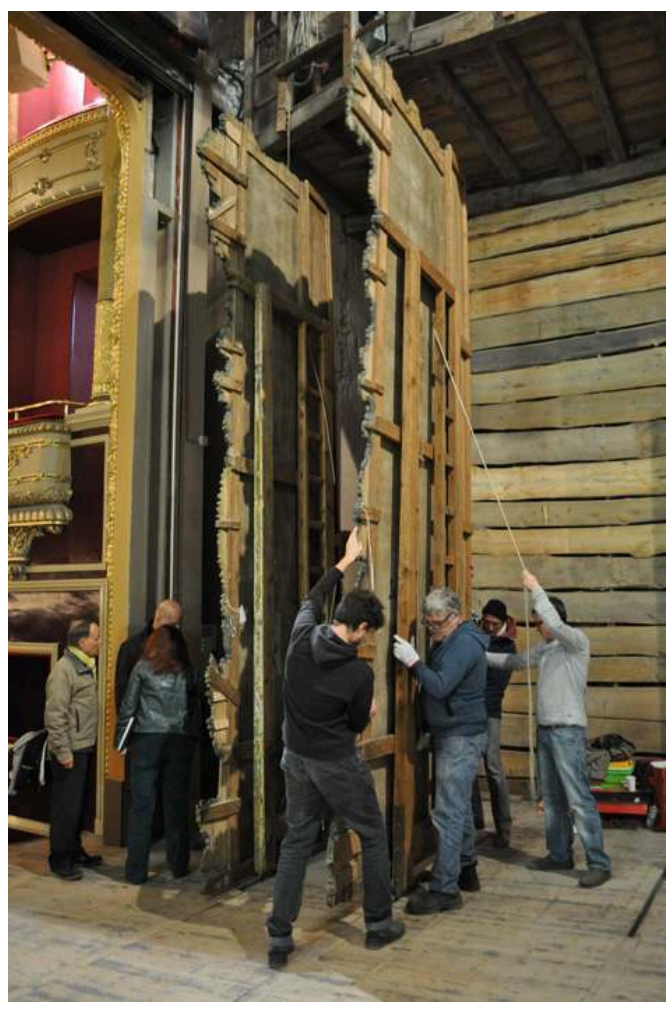

Théâtre de Châtellerault, Vienne. La formation des machinistes à la manipulation s'est achevée par l'implantation sur scène de l'ensemble Forêt : ici le guindage d'un châssis de coulisse sur un faux châssis.

Phot. Embs, Anne. (C) DRAC Poitou-Charentes, novembre 2013. $\mathrm{du}$ XIX ${ }^{e}$ siècle encore en usage ont été significativement réduits grâce à une formation à la manipulation des décors et de la machinerie proposée aux machinistes de ce «musée vivant », formation qui leur a permis d'intégrer une démarche conservatoire dans leurs pratiques professionnelles.

Mais, au-delà de ces mesures préventives qui prennent tout leur sens dans leur application aux ensembles patrimoniaux, les projets et les interventions de restauration retenus et présentés au fil des articles attestent une finalité partagée : la restitution de l'aspect visuel initial, même, s'il le faut, au prix d'un subterfuge illusionniste qui préserve l'esprit des lieux.

Ainsi, les tentures relevant de l'ensemble que constitue le Salon jaune, réservé à l'impératrice Joséphine à Fontainebleau, ont été maintenues en place malgré leur décoloration irréversible. Elles ont retrouvé leur intensité chromatique et l'élégance de leurs motifs brodés d'origine grâce à la pose d'une crêpeline colorée et sérigraphiée produisant l'illusion optique attendue.

Cet objectif de restitution d'un état initial, parfois perdu, a également fondé le choix d'intégrer à certains ensembles originaux des copies remplaçant des éléments disparus ou trop dégradés pour faire l'objet d'une restauration. Ainsi, les vêtements des mannequins peuplant les dioramas du Museon Arlaten ont fait l'objet de copies d'évocation réalisées à partir de textiles imprimés numériquement, tandis que le 
luminaire d'applique manquant dans le hall de la maison Cavrois a été reproduit sur le même modèle que les trois autres pour restituer la symétrie décorative d'origine.

La recherche d'un équilibre toujours incertain entre conservation et accueil du public donne lieu à un large éventail de mesures conservatoires et curatives qui, associées, permettent de maintenir ces ensembles in situ malgré les limites explicitées par les auteurs.

32 Le choix de réguler et de limiter le nombre de visiteurs constitue une constante dans la préservation de ces ensembles; il a parfois été assorti de mesures visant à limiter vols et dégradations. Dans le cas des écoles nationales d'horlogerie de Besançon ou d'optique de Morez, celles-ci se résument à suspendre au moins temporairement, faute de protection suffisante, la cohésion de l'ensemble (bâtiments et matériel pédagogique) en conservant certains objets ex situ. Au musée Nissim de Camondo (Paris), un choix semblable a été fait en mettant en réserve les objets les plus petits ou les plus accessibles aux visiteurs et en roulant partiellement les tapis risquant une usure rapide en raison d'une fréquentation fort éloignée de celle qui existait à son ouverture. À Mirecourt (Vosges), dans l'atelier du luthier Lucien Gérome, un ensemble dont les éléments ont été hiérarchisés dans leur statut dès l'acquisition, certains matériaux d'étude contribuant au décor ont été dupliqués et peuvent sans inconvénient majeur être détériorés ou subtilisés par les visiteurs.

Figure 6

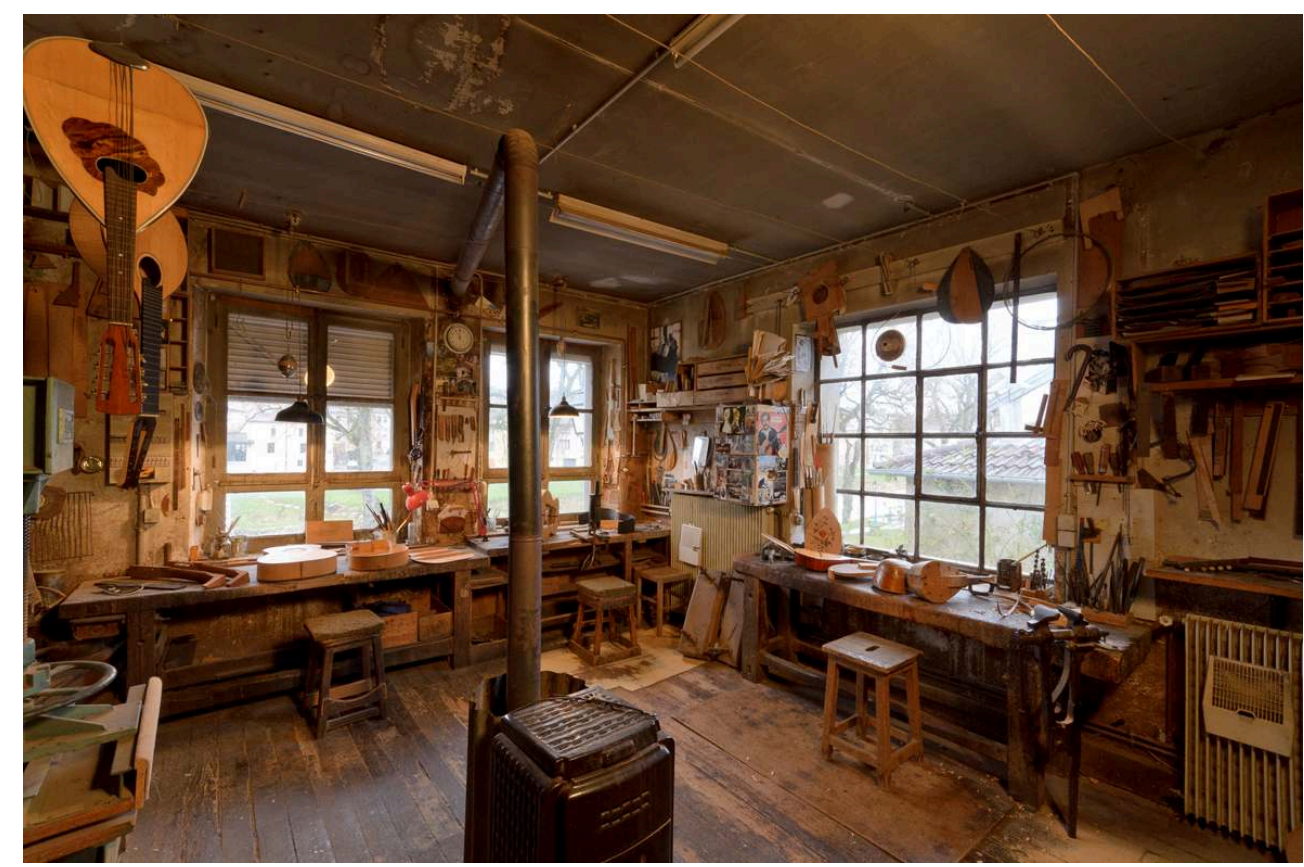

Atelier de luthier à Mirecourt, Vosges. La salle dite « l'atelier ». Atelier Gérôme.

Phot. PHILIPPOT, CLAUDE, 2011. (c) Musée de la lutherie.

Quant aux programmes didactiques et aux outils pédagogiques qui accompagnent l'ouverture au public des ensembles présentés dans ce numéro, ils s'inscrivent dans la continuité des expériences de médiation menées à partir des années 1970 dans les écomusées et sur les sites industriels en cours de patrimonialisation. Ils se répartissent entre visites commentées et démonstrations techniques mais s'enrichissent de cette notion d'ensemble qu'il s'agit de transmettre également au public. 
34 Ainsi, dans les " maisons d'illustres », celle de Camille Flammarion, de Léonard Foujita ou d'Antoine Bourdelle, dans les musées abritant des ensembles reconstitués, comme dans l'ancien MNATP ou au musée d'Art et d'Histoire de Saint-Denis, les médiateurs explicitent par leur commentaire ou grâce à des méthodes participatives ce qui reste souvent invisible et difficile à appréhender par le public: la mémoire d'un homme, d'une famille ou d'une communauté professionnelle.

Pour les ensembles relevant d'une activité artisanale ou industrielle révolue, dans une convention tacite établie depuis des décennies toujours attendue par le public, la médiation prend la forme, comme à Mirecourt ou dans les scieries vosgiennes, d'une démonstration impliquant alors l'usage temporaire de certaines machines dont la conservation se trouve ainsi améliorée.

Mais quelques nouveaux outils didactiques numériques plus originaux, à l'état de projet ou déjà réalisés, témoignent de l'évolution des méthodes de restitution au public. Ils ouvrent de nouvelles pistes à la médiation d'ensembles riches de leur complexité sémantique. Ainsi, la reconstitution virtuelle actuellement en cours de la cage de Faraday et de divers appareillages pour rendre au générateur Cockcroft-Walton installé au fort de la Vitriolerie (Lyon) son aspect initial, ou le dispositif numérique permettant la manipulation virtuelle des instruments scientifiques liés aux travaux de Marie Curie présentés autour de son bureau/laboratoire constituent-ils des exemples intéressants de transmission interactive d'informations denses et diversifiées. À moyen terme, sous réserve d'une complexification, ce type d'outil devrait apporter une aide significative au visiteur non seulement pour saisir ce qui sous-tend ces ensembles, qu'ils soient mobiliers, industriels ou techniques, mais aussi pour se les approprier dans une expérimentation toujours féconde croisant concept, technique et documentation.

\section{Échecs et reconquêtes}

37 Comment répondre à la volonté d'un propriétaire qui souhaite le maintien in situ et la conservation de l'ensemble constitué par ses ancêtres ou ses prédécesseurs? Des exemples des difficultés rencontrées et des solutions apportées ou encore des échecs patrimoniaux illustrent le chemin difficile vers la transmission. L'administration est régulièrement confrontée à la question du maintien des protections juridiques pesant sur les ensembles dispersés ou la fermeture au public des lieux patrimoniaux. Dans certains cas, les pertes sont irrémédiables. Dans le domaine du patrimoine privé, les échecs sont connus et souvent dénoncés par les associations de sauvegarde ou les juristes spécialisés ${ }^{3}$.

39 Nous souhaitions voir évoquer comme autant de leçons pour l'avenir ces exemples négatifs. Ce chapitre intitulé "Échecs et reconquêtes" présente en conséquence des dispersions sans doute définitives comme la pharmacie néo-égyptienne de ClermontFerrand (Puy-de-Dôme), cas emblématique de la difficulté de pérenniser un ensemble lié à une fonction commerciale, ou des réflexions sur les risques et les difficultés du maintien d'un ensemble scientifique tel que celui conservé à l'observatoire Camille Flammarion, du fait de la multiplicité des acteurs en présence. La question de l'avenir des unités écologiques conçues pour le musée national des Arts et Traditions populaires est aussi posée. Enfin, les collections du comte de Chambord, maintenant bien identifiées, seront-elles mises en valeur pour elles-mêmes? 
Figure 7

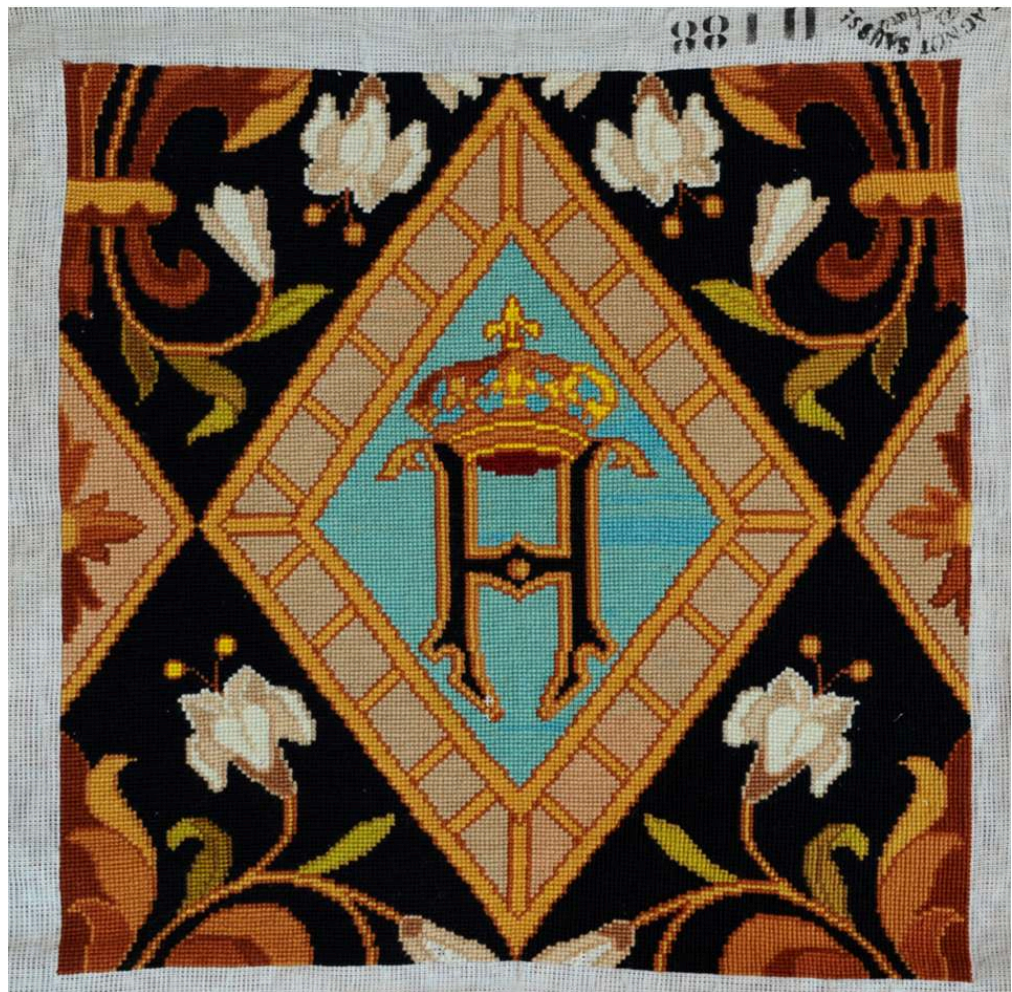

Pièce de broderie au point Manufacture Gagnot-Sausse, vers 1871/1880. Domaine national de Chambord, fonds ancien.

(c) Domaine national de Chambord.

À ces cas problématiques répondent des exemples de reconstitutions d'ensembles dispersés. Il importait de montrer comment la conjonction des bonnes volontés et de la prise de conscience de l'intérêt patrimonial peut contribuer à maintenir des ensembles dans les lieux pour lesquels ils ont été conçus, voire à reconstituer des ensembles dispersés. Chacun de ces cas, qu'il s'agisse de la commande publique de Jean Zay ressuscitée, de la villa Cavrois remeublée ou de la restauration de la chapelle des Pénitents blancs de Villeneuve-sur-Lot, répond à un projet scientifique bien spécifique mais fondé sur un état historique de référence bien documenté. 


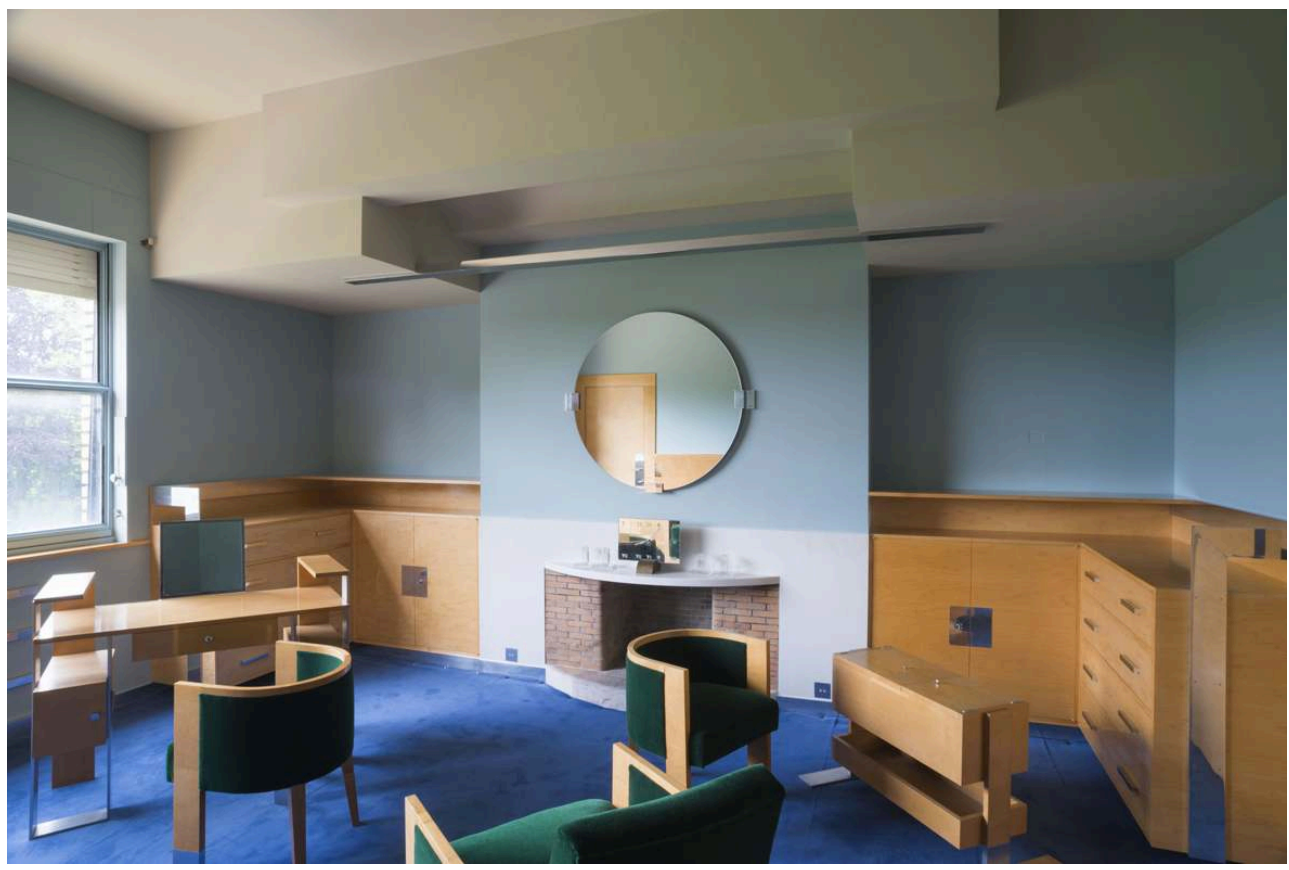

Croix, Nord. Villa Cavrois, le boudoir remeublé.

Phot. Ballot, Jean-Christophe/Centre des monuments nationaux. (c) Jean-Christophe Ballot, 2015.

41 À cette première série d'articles ici réunis viendront s'ajouter les actes des journées d'étude «Mobiliers, ensembles, décors - Conserver, restaurer, faire vivre » qui ont eu lieu les 28 et 29 janvier 2016 à l'auditorium de la Galerie Colbert (Paris) ${ }^{4}$. Ces journées étaient consacrées à la conservation et à la restauration des ensembles mobiliers cohérents, quand le contenant architectural est en adéquation avec le contenu, mobilier et décor. Ces journées d'étude, suivies par plus de 250 personnes, ont fait l'objet d'une captation audio d'ores et déjà mise en ligne par l'Institut national du patrimoine. De même est accessible un dossier présentant de manière détaillée le programme, des bibliographies de référence et des extraits de glossaire utiles à la compréhension de l'évolution du concept d'ensemble ${ }^{5}$.

Plusieurs contributions ont été faites à plusieurs voix, dans une volonté affirmée d'interdisciplinarité. Les articles issus des thèmes traités lors de ces journées d'étude permettront d'enrichir en particulier les chapitres de ce numéro évoquant les questions de conservation-restauration et de présentation au public. Il s'agit de s'interroger sur les partis à prendre en termes d'étude, de recherche, de traitements de conservation, de projets de restauration voire de restitution dans ces ensembles complexes, en étudiant les différentes méthodologies mises en œuvre lors d'expériences récentes, tant dans les musées que dans les monuments historiques, les demeures ou les édifices religieux. Certaines interventions évoquent les nouveaux outils de médiation, qu'il s'agisse de reconstitutions virtuelles d'ensembles historiques, de visites en ligne de lieux difficiles d'accès ou de l'utilisation de l'éclairage, de la musique ou plus largement, des sons, pour rendre encore plus vivants ces ensembles mobiliers.

La coordination de la rédaction de ce numéro d'In Situ a été confiée à Judith Kagan, conservateur général du patrimoine (service du Patrimoine, sous-direction des 
Monuments historiques et des Espaces protégés) et Anne Tricaud, conservateur en chef du patrimoine (service des Musées de France, sous-direction des Collections) en lien avec Dominique Séréna-Allier, directrice du Museon Arlaten en Arles.

\section{Exemples récents de protection au titre des monuments historiques}

Voir le site : http://www.culture.fr/Ressources/Moteur-Collections

Les usines Sommer (Ardennes)

Château de La Rochepot (Côte-d'Or)

Collection Napoléon au château de Cendrier (Dordogne)

Maison de Champollion (Isère)

Maison de Louis Carré à Bazoches (Yvelines)

Château de Vaux-sur-Seine (Yvelines)

Château de Breteuil à Choisel (Yvelines)

Collections universitaires (Montpellier, herbiers...)

Cabinets de physique des lycées (Dijon, Bar-le-Duc, Moulins, Périgueux, Versailles...)

Collections scientifiques (observatoires, École polytechnique)

Collections hospitalières (Dijon, Roanne, Château-Thierry, Lyon, Toulouse...)

Exemples malheureux de dispersions d'objets (protégés ou non), de déclassement d'objets...

L'usine Sidoux, métiers à tisser guipure (Aisne)

Les papeteries d'Angoulême (Charente)

La manufacture Le Manach à Tours (Indre-et-Loire)

L'hôtel particulier Follot à Paris (Paris)

L'hôtel du Jouhannel de Jenzat à Riom (Puy-de-Dôme)

Les métiers à tisser de Villeurbanne (Rhône)

Le château de Canteleu (Seine-Maritime)

Le château de La Roche-Guyon (Val d'Oise)

L'hôtel de Saint-Priest d'Urgel à Avignon (Vaucluse)

Le château de Dampierre-en-Yvelines (Yvelines)

Exemples de restaurations et de nouvelles présentations

Maison Mantin à Moulins (Allier), musée de France

Salon de musique du château de Compiègne (Oise), MH, musée de France

Hôtel Sabatier d'Espeyran à Montpellier (Hérault), MH, musée de France

Château d'Azay-le-Rideau (Indre-et-Loire), MH, CMN

Château d'Espeyran (Gard), MH

Hôtel de Lunas à Montpellier (Hérault), MH, CMN

L'Harmas, maison de Jean-Henri Fabre à Sérignan-du-Comtat (Vaucluse), MNHN, MH

Maison de Pasteur à Dôle (Jura), MH, musée de France

Musée du Louvre, département des Objets d'art, salles du XVIII ${ }^{\mathrm{e}}$ siècle, musée de France

Musée des Arts décoratifs, period rooms, musée de France 
Château de Malmaison (Hauts-de-Seine), MH, musée de France

Château de Versailles (Yvelines), MH, musée de France, etc.

\section{Politique de valorisation}

Réseau des maisons des illustres: http://www.culturecommunication.gouv.fr/Aidesdemarches/Protections-labels-et-appellations/Label-Maisons-des-Illustres

\section{NOTES}

1. - Voir KAGAN, Judith, SCHOENSTEIN, Frantz et PIEL, Caroline. «Pour résoudre une lacune de la loi du 31 décembre 1913 relative aux monuments historiques : "Ensembles historiques mobiliers, servitude de maintien in situ" ». Actes des journées d'étude de l'association des conservateurs des antiquités et objets d'art "Regards sur le mobilier domestique». Dijon, 3 octobre 2014. Arles : Actes Sud, 2015, p. 117-128. Voir aussi les nombreux exemples donnés dans l'étude d'impact du PLCAP sur le site de l'Assemblée nationale: Ensembles historiques mobiliers et servitude de maintien dans les lieux: http://www.assemblee-nationale.fr/14/projets/pl2954ei.asp\#P4351_543657 [consulté le 21/07/2016].

2. - Ce numéro d'In Situ devrait être mis en ligne au deuxième semestre de 2016.

3. - La sauvegarde du patrimoine mobilier - Position de la Société pour la protection des paysages et de l'esthétique de la France (SPPEF): http://www.sppef.fr/category/nos-combats/08-lasauvegarde-du-patrimoine-mobilier/ [consulté le 21/07/2016]. LACAZE, Julien. «Le démantèlement des grandes demeures : de La Roche-Guyon à Dampierre (1987-2013)», 2013 : http://www.latribunedelart.com/le-demantelement-des-grandes-demeures-de-la-roche-guyona-dampierre-1987-2013 [consulté le 21/07/2016]. LACAZE, Julien. "Patrimoine contextuel et ensembles mobiliers : enfin une loi, Sites \& Monuments », n²21, 2014 : http://www.sppef.fr/wpcontent/uploads/2015/09/sppef_patrimoine-contextuel-et-ensembles-mobilierssitesmonuments-n\%C2\%B0-221-2014.pdf [consulté le 21/07/2016].

4. - Colloque organisé par la direction générale des Patrimoines (DGP), service des Musées de France (SMF) et service du Patrimoine (SP), en partenariat avec l'Institut national du patrimoine (Inp), le Centre de recherche et de restauration des musées de France (C2RMF), le Centre des monuments nationaux (CMN), le Mobilier national et le Château de Versailles.

5. - Voir le site : http://mediatheque-numerique.inp.fr/Colloques/Mobiliers-ensembles-decors.Conserver-restaurer-faire-vivre [consulté le 21/07/2016]. Résumés des interventions Bibliographie - Terminologie. Dossier documentaire réalisé par l'Inp et les représentants du comité scientifique, Judith Kagan, Lucile Montagne et Bruno Saunier. 


\section{AUTEURS}

\section{JUDITH KAGAN}

Conservateur général du patrimoine, service du Patrimoine, sous-direction des Monuments historiques et des Espaces protégés judith.kagan@culture.gouv.fr

\section{DOMINIQUE SÉRÉNA-ALLIER}

Directrice du Museon Arlaten, Arles dominique.serenaallier@cg13.fr

ANNE TRICAUD

Conservateur en chef du patrimoine, service des Musées de France, sous-direction des Collections anne.tricaud@culture.gouv.fr 